\title{
Survival strategies of cancerous cells: a novel perspective
}

\author{
Sachin C Sarode*,1, Nilesh Kumar Sharma², Gargi S Sarode ${ }^{1}$, Pritish Nilendu ${ }^{3}$ \& \\ Shankargouda Patil ${ }^{4}$ \\ ${ }^{1}$ Department of Oral Pathology \& Microbiology, Dr DY Patil Dental College \& Hospital, Dr DY Patil Vidyapeeth, Maheshnagar, \\ Pimpri, Pune - 18, Maharashtra, India \\ ${ }^{2}$ Cancer \& Translational Research Lab, Dr DY Patil Biotechnology \& Bioinformatics Institute, Dr DY Patil Vidyapeeth, Pune - \\ 411033, Maharashtra, India \\ ${ }^{3}$ Cancer \& Translational Research Lab, Department of Biotechnology, Dr DY Patil Biotechnology \& Bioinformatics Institute, Pune - \\ 411033, Dr DY Patil Vidyapeeth, Pune, Maharashtra, India \\ ${ }^{4}$ Department of Maxillofacial Surgery \& Diagnostic Sciences, Division of Oral Pathology, College of Dentistry, Jazan University, \\ Jazan - 45142, Saudi Arabia \\ *Author for correspondence: Tel.: +91 992249 2465; Fax: +97 20 27423427; drsachinsarode@gmail.com
}

\section{"We believe that as a survival tactic, cancerous cells release certain systemic factors that prevent the development of malignancies at other sites of the body."’}

First draft submitted: 8 August 2018; Accepted for publication: 16 August 2018; Published online: 7 September 2018

Keywords: cachexia $\bullet$ carcinogenesis $\bullet$ cell survival $\bullet$ targeted therapy $\bullet$ tumorigenesis

Although a reality, the co-existence of two histogenetically different neoplasms in a single body is a rare event. This enigmatic question of 'rarity' has never been addressed in the literature. We believe that as a survival tactic, cancerous cells release certain systemic factors that prevent the development of malignancies at other sites in the body. These factors can also influence normal tissues of the body, leading to a well-known phenomenon called 'cancer cachexia'. Efforts were made to support the generated hypothesis by discussing established facts regarding cancer cachexia, epigenetic changes and cellular cannibalism. This hypothesis may contribute to the understanding of new classes of factors secreted from tumors and may present new therapeutic opportunities in treating cancer (Figure 1).

All the cells in the body have the potential for malignant transformation, and cancer can thus occur in any organ anywhere in the body. The transformation of a normal cell to a malignant cell is a sequential process, attributed mainly to environmental-induced mutations, that involves a myriad of molecular events triggering numerous signaling pathways. These signaling pathways affect transformation-relevant biological processes, including survival, proliferation, invasion, migration and angiogenesis. The completely established malignancy is essentially composed of transformed cells that share histogenetically similar origin. It is generally observed that development of totally different malignancy at distant site and different tissue origin is extremely rare event. Why would there be predominance of 'one person one malignancy' rule in carcinogenesis?

This research question has not been deliberated in the literature. We believe that as a survival tactic, cancerous cells release certain systemic factors that prevent the development of malignancies at other sites of the body. These factors can also influence normal tissues of the body, leading to the well-known phenomenon called 'cancer cachexia'.

Cancer cachexia is characterized by secretion cachexins such as chemokines, cytokines and other soluble extracellular proteins/peptides to maintain their won favorable niche [1,2]. These cachexins have been suggested to have role of antigrowth, antimetabolic adaptation and antiphysiological survival effects. There is sufficient evidence indicating that several paracrine signaling molecules secreted from the primary tumor microenvironment may be key signals that modulate the intracellular signaling cascades crucial for growth and survival of the target cells, including muscle cells and adipose tissue $[1,2]$. Therefore, it seems logical to propose that these antigrowth and metabolic 


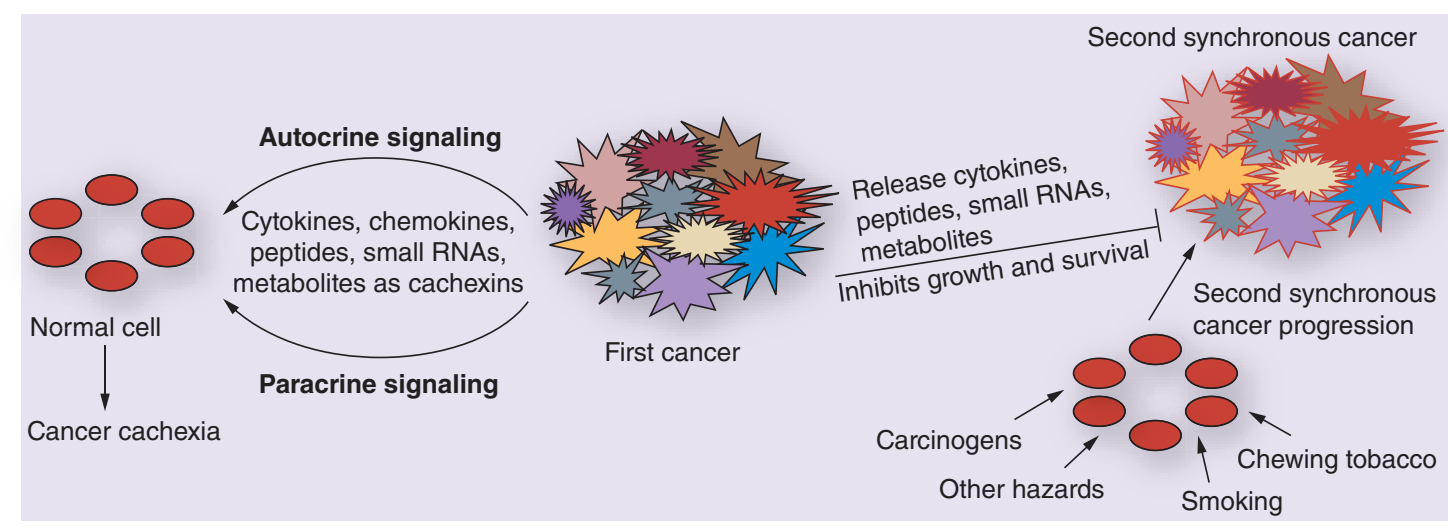

Figure 1. Role of paracrine molecules in cancer cachexia and occurrence of second synchronous cancer.

starvation effects achieved through paracrine signaling may be responsible for suppressing the development of histogenetically different second primary malignancy in a single cancer patient.

In the well-known phenomenon of cancer cachexia, specifically cancer-associated muscle cell catabolism, the possible role of epigenetic factors is not ruled out [3,4]. The epigenetic microenvironment created by one type of primary cancer type may inhibit growth, proliferation as well as the chance of second malignancy. Based upon the probability of genetic alterations, the incidences of multiple synchronous cancers should be more frequent at the intracancer patient level, but the metabolic adaptation strategies driven by epigenetic factors within the tumor microenvironment may explain the inhibition of a second malignancy in a single patient.

In a population-based study using the Swedish Family Cancer Database, there was an assumption that the probability of colorectal adenoma was higher in the offspring and siblings of patients with endometrial, prostate and stomach cancer and leukemia and in the siblings of patients with pancreatic cancer and multiple myeloma [5]. Such correlation among cancer patients can be explained merely based on genetic events and a game of chance. Despite the correlations found in this large population study, reports of more than one type of cancer in the same patient synchronously seem to be rare, and the lack of such incidences cannot be explained by the probability of genetic mutations alone. Rather, the extra-genetic landscape may be responsible for the observations of one type of primary malignancy occurring at a time, excluding the cases of the development of malignancy after therapeutic events or immune modulation.

An additional aspect to the present hypothesis is that the rare occurrence of histogenetically second tumor may be linked to immune modulation strategies adopted by tumor cells. There is strong evidence showing that during tumor development, cancer cells are able to create their niches in such a way that results in many immune cells supporting cancer growth and proliferation. If this phenomenon is true, then it may be possible to use these immune cells and their secreted molecules, such as cytokines, peptides and small RNAs, to hijack the physiological systems of the entire human body, which may inhibit another primary tumor from forming in the same patient. There is a chance that once one type of primary cancer is able to establish its niches in a patient, those tumor cells, along with pro-tumor, noncancerous cells, may be able to manufacture and secrete a set of anti-inflammatory cytokines that inhibit other cancer types in the same patient.

In a century-old paper by Bugher [6], the author suggested that the actual occurrence of multiple cancers in the same patient appeared to be exceedingly low, considering the probability of genetic alterations and the reasons for this could lie beyond the genetic code. In that study, the author analyzed a large collection of data from cancer patients and reached conclusions on cancer occurrence, but the data did not distinguish between synchronous or asynchronous cancers.

Here, the authors would like to add to the discussion regarding a genetic study carried out by Michiels et al. [7], which suggests that certain types of gene polymorphisms, such as those in DNA repair genes, could be correlated to the simultaneous occurrence of lung cancer and head and neck cancer. However, the possibility that DNA repair gene polymorphisms are present in the same patient simultaneously cannot be excluded. Despite that possibility, synchronous occurrences of lung cancer and head and neck cancer have been reported to be rare. Therefore, these observations cannot be explained by the probability of genetic mutation alone; instead, they should be viewed in 
terms of extra-genetic, intrapatient molecular signaling events occurring within the tumor niches and outside of the tumor niches, which can modulate immune responses, cancer cachexia and other components to build other distinct types of tumor niches.

In a rare clinical study, it was reported that approximately $14 \%$ of studied patients with primary neuroendocrine tumors had a synchronous or meta-synchronous second primary malignancy in the colon, breast or bladder [8]. Another study reported by Verstovsek et al. [9] and Agarwal [10] on synchronous primary cancers of the breast and cervix suggested that such cases are unusual in nature. In these rare studies on neuroendocrine tumors, those tumors are widely accepted to be a rare form of malignancy, and the authors have not suggested any molecular mechanisms. However, we infer that in the case of neuroendocrine tumors, distinct characteristics of the tumor microenvironment may be acting as procancer signals and may favor the synchronous and metasynchronous existence of a second primary cancer.

Data from Globocan 2008 and 2012 on various cancer incidence trends in India [11] showed that cervical cancer was the most common cancer in women in India. This was the scene for almost four decades (or more). But over the last 10 years or so, breast cancer has been rising steadily, and for the first time now, breast cancer is the most common cancer in women in India, way ahead of cervical cancer. It has been stated that this could be due to a decrease in the incidence of cervical cancer. But most of it will be because of a rapid rise in the numbers of breast cancer cases. In context to proposed hypothesis, the decreased incidence rate of cervical cancer could be due to increased incidence of breast cancer in females. The existence of breast cancer in a patient is avoiding occurrence of any other cancer in the body, in this instance its cervical cancer. Hence, just like a cause and effect relationship, as breast cancer incidence is increased, there is decrease in cervical cancer decreases over a period of time. There could be no role of human papillomavirus vaccine or screening test for bringing down the incidence rate of cervical cancer in India. To support this, a systematic review of pre- and post-licensure trials of the human papillomavirus vaccine by a Canadian team shows that its effectiveness is not only overstated (through the use of selective reporting or 'cherry picking' data) but also completely unproven [12].

\section{Conclusion}

Survival is an important hallmark of carcinogenesis. In the present paper, a new survival strategy has been put forward that explains the rarity in the development of histogenetically different neoplasms at secondary sites. We believe that as a survival tactic, cancerous cells release certain systemic factors that prevent the development of malignancies at other sites in the body. Attributing such behavior of cancer cell to the extremely high nutritional demand is quite conceivable. It is recommended to investigate the aforementioned novel hypothesis as it holds tremendous potential for development of targeted therapy in future.

\section{Future perspective}

To investigate this proposed hypothesis, a 3D tumor tissue model is suggested for the synchronous maintenance and growth of two different cancer types. In this model, cutting-edge tools, such as proteomics, transcriptomics, metabolomics and small RNA Seq technology could be used to reveal synergistic or antagonistic effects from one type of carcinoma to another. Additionally, in vitro cell culture studies could be designed to investigate the effect of extracellular secreted factors from a certain type of cancer on established normal and cancerous primary cell lines. An in vivo animal model study could explore the participating molecular and cellular factors to better understand crosstalk between synchronous tumors of different types.

If this proposed hypothesis is supported with the aforementioned experiments, it may reveal novel therapeutic opportunities for cancer management. Such research may provide evidence beyond the existing knowledge about factors secreted by cancer cells that create aberrant signaling, promoting cancer hallmarks, including invasiveness, metastasis, angiogenesis and cancer cachexia. Identifying these factors, including peptides, proteins, metabolites, small RNAs, mRNA and circulating DNA from the tumor microenvironment, may provide prognostic markers. Apart from the use of these factors in determining prognosis, blocking or removing these factors within the tumor microenvironment using a new class of precision drugs, such as peptide mimetics, RNA mimetics, small RNA inhibitors and new class of epigenetic modifiers, may help to reduce the cancer cachexia phenomena and cancerrelated mortality. Knowledge of the structure and nature of growth-inhibitory factors from a given cancer type could be translated into a new class of anticancer drugs and approaches against other cancer types. Similar to the existing idea of bacterial toxins from one bacterium acting upon another bacterium, this hypothesis has the potential for the development of a new class of toxins derived from one type of cancer to act upon another cancer. 
Hypothetically, in interpatient therapeutic interventions, it may be possible to extract derived extracellular factors from a patient with one cancer type and deliver them to a patient with a different cancer type; one type of cancer may act as the enemy of another cancer.

\section{Financial \& competing interests disclosure}

The authors have no relevant affiliations or financial involvement with any organization or entity with a financial interest in or financial conflict with the subject matter or materials discussed in the manuscript. This includes employment, consultancies, honoraria, stock ownership or options, expert testimony, grants or patents received or pending, or royalties.

No writing assistance was utilized in the production of this manuscript.

\section{References}

1 Zhang G, Liu ZI, Ding H et al. Tumor induces muscle wasting in mice through releasing extracellular Hsp70 and Hsp90. Nat. Commun. 8,589 (2017).

2 Alves MJ, Figuerêdo RG, Azevedo FF et al. Adipose tissue fibrosis in human cancer cachexia: the role of TGF $\beta$ pathway. BMC Cancer 17, 190 (2017).

3 Schäfer M, Oeing CU, Rohm M et al. Ataxin-10 is part of a cachexokine cocktail triggering cardiac metabolic dysfunction in cancer cachexia. Mol. Metab. 5, 67-78 (2016).

4 Carr RM, Enriquez-Hesles E, Olson RL, Jatoi A, Doles J, Fernandez-Zapico ME. Epigenetics of cancer-associated muscle catabolism. Epigenomics doi:10.2217/epi-2017-0058 (2017) (Epub ahead of print).

5 Hiripi E, Bermejo JL, Sundquist J, Hemminki K. Association of colorectal adenoma with other malignancies in Swedish families. Br. J. Cancer 98, 997-1000 (2008).

6 Bugher JC. The probability of the chance occurrence of multiple malignant neoplasms. Am. J. Cancer 21, 809-824 (1934).

7 Michiels S, Danoy P, Dessen P et al. Polymorphism discovery in 62 DNA repair genes and haplotype associations with risks for lung and head and neck cancers. Carcinogenesis 28, 1731-1739 (2007).

8 Prommegger R, Ensinger C, Steiner P, Sauper T, Profanter C, Margreiter R. Neuroendocrine tumors and second primary malignancy - a relationship with clinical impact? Anticancer Res. 24, 1049-1051 (2004).

9 Verstovsek S, Verschraegen CF, Edwards CL et al. Synchronous primary cancers of the breast and cervix: planning multidisciplinary primary treatment [clinico-pathological conference]. Am. J. Clin. Oncol. 23, 99-103 (2000).

10 Agrawal R. Synchronous dual malignancy: successfully treated cases. J. Cancer Res. Ther. 3, 153-156 (2007).

11 Breast Cancer India. Statistics of breast cancer in India: global comparison. (2017). www.breastcancerindia.net/statistics/stat_global.html

12 Tomljenovic L, Spinosa JP, Shaw CA. Human papillomavirus (HPV) vaccines as an option for preventing cervical malignancies: (how) effective and safe? Curr. Pharm. Des. 19(8), 1466-1487 (2013). 